\title{
Coronary physiology and percutaneous intervention managed with gadolinium road mapping and intravascular ultrasound in hyperthyroidism
}

\author{
Peter Tajti ${ }^{1,2 *}$, Mohamed Ayoub ${ }^{1 *}$, Thomas Nuehrenberg ${ }^{1}$ Kambis Mashayekhi $^{1}$ \\ ${ }^{1}$ Department of Interventional Cardiology, Cardiology and Angiology II, \\ University Heart Center Freiburg - Bad Krozingen, Germany \\ ${ }^{2}$ Gottsegen György Hungarian Institute of Cardiology, Budapest, Hungary
}

\section{Introduction}

The incidence of iodine-induced thyrotoxicosis after the administration of iodinated contrast agent remains low in various patient cohorts $(0.2 \%$ to $1.9 \%[1,2])$. In patients with coexisting subclinical hyperthyroidism and ischemic heart disease, however, contrast administration may provoke or worsen its clinical condition requiring additional endocrinology treatment and clinical follow-up after the initial coronary angiography (CAG) or percutaneous coronary intervention (PCI) [3]. Alternative contrast agents - such as gadolinium - replacing iodinated contrast media during coronary angiography have been introduced previously in small cohorts and case reports to avoid nephrotoxicity [4, 5], contrast allergy [6], or iodinated-contrast-induced thrombocytopenia [7]. The present study, describes a case using a low amount of gadolinium for coronary anatomy reconstruction assisting the coronary physiology and imaging guided $\mathrm{PCI}$ in an elderly patient with acute coronary syndrome (ACS) who was simultaneously diagnosed with hyperthyroidism.

\section{Case report}

An 85-year-old-man, with a history of coronary artery disease (CAD), was referred to the documented center with severe ongoing chest pain and progressive dyspnea. The patient had a prior PCI of the right coronary artery and distal left circumflex artery (LCX) performed 1 year prior to the current admission to hospital. Additionally, the patient had severe peripheral artery disease and had undergone multiple prior percutaneous transluminal angioplasty of the limbs. Relevant coronary risk factors included hypertension, hypercholesterinemia and positive family history of CAD. The patient was hemodynamically stable, and on physical examination no relevant abnormalities were detected. Initial laboratory testing revealed an elevated level of high sensitive troponin $\mathrm{T}$ and a normal level of creatine kinase (CK), and creatine kinase myocardial band (CK-MB). An electrocardiogram showed no relevant ST segment changes, and transthoracic echocardiography showed normal biventricular function (left ventricular ejection fraction of 55\%) without significant valve disease. The estimated glomerular filtration rate was $74 \mathrm{~mL} / \mathrm{min} / 1.73 \mathrm{~m}^{2}$. Additionally, thyroid stimulating hormone was suppressed, $0.11 \mu \mathrm{lU} / \mathrm{L}$ (normal range [NR] $0.27-4.2 \mu \mathrm{lU} / \mathrm{L}$ ), whereas $\mathrm{T}_{3}$ (4.2 pmol/L [NR 3.1-6.8 pmol/L]) and $\mathrm{T}_{4}$ was normal (16.7 pmol/L [NR $12-22 \mathrm{pmol} / \mathrm{L}])$. Due to the possibly underlying subclinical hyperthyroidism, an endocrinological consultation took place which revealed the first diagnosis of an ongoing thyroid autonomy.

A decision was made to proceed with invasive CAG within 72 hours due to intermediate risk, using low-dose gadolinium for anatomy road mapping.

Address for correspondence: Kambis Mashayekhi, MD, Deputy Medical Director - Director of CTO and High Risk Angioplasty, Interventional Cardiology Department, Cardiology and Angiology II, University Heartcenter Freiburg — Bad Krozingen, Südring 15, 79189 Bad Krozingen, Germany, e-mail: kambis.mashayekhi@universitaets-herzzentrum.de Received: 15.06.2020

*P. Tajti and M. Ayoub contributed equally to this manuscript.

This article is available in open access under Creative Common Attribution-Non-Commercial-No Derivatives 4.0 International (CC BY-NC-ND 4.0) license, allowing to download articles and share them with others as long as they credit the authors and the publisher, but without permission to change them in any way or use them commercially. 


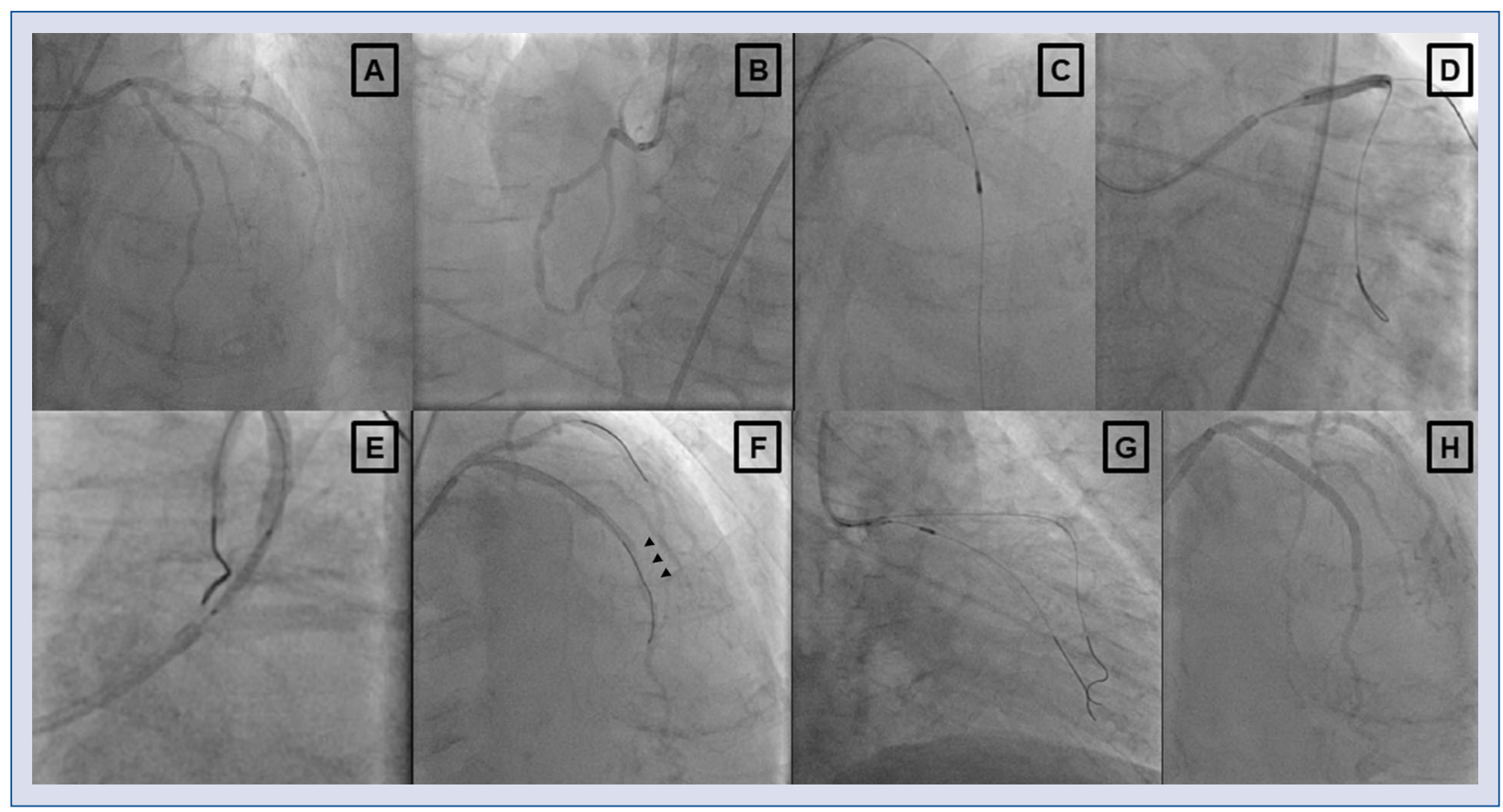

Figure 1. Percutaneous coronary intervention road mapping with intra-arterial gadolinium injection assisting fractional flow reserve coronary physiology assessment and intravascular ultrasound (IVUS) guided stent implantation; A, B. Left and right coronary injections with gadolinium; C. IVUS in the left anterior descending artery (LAD); D, E. Predilation of the proximal LAD and stenting; F. Distal edge dissection (arrowhead); G. IVUS in the left circumflex artery; $\mathbf{H}$. Final result.

According to a previously reported risk of cardiac arrhythmia and potential hemodynamic instability [8], $5 \mathrm{mg}$ of metoprolol intravenously (relative beta-1 selectivity) was administered, defibrillator paddles were applied, hemodynamics were continuously measured and 1-2 cc first time injections before angiography of the left and right coronaries were administered (Fig. 1A, B). Coronary physiology assessment of fractional flow reserve (FFR) (Comet pressure wire, Boston Scientific, Natick, Massachusetts, USA) was performed. At maximum hyperemia, FFR of the left anterior descending artery (LAD) was positive (0.77), whereas LCX remained functionally insignificant (0.89). Thereafter proceeding with PCI, exchanging for regular workhorse wire (Sion Blue [Asahii Intecc. Nagoya, Japan]) and evaluating the plaque burden, vessel diameter and lesion length (Fig. 1C) with intravascular ultrasound (IVUS [Eagle Eye, Philips Volcano, Amsterdam, Netherlands]). Two drug eluting stents were implanted $(2.5 \times 12 \mathrm{~mm}$, $2.75 \times 38 \mathrm{~mm})$ in the middle and proximal LAD segments. After post-dilation optimal stent expansion was confirmed with control IVUS (Fig. 2). Total procedure and fluoroscopy times were 55 and 10 minutes, respectively. $35 \mathrm{~mL}$ of gadolinium was used overall. The patient was discharged on dual antiplatelet therapy on the $4^{\text {th }}$ day post-PCI. Endocrinology control was scheduled to evaluate the thyroid autonomy 2 weeks post-PCI, and thiamazole was added to his therapy at discharge.

\section{Discussion}

This report illustrates the concept of using gadolinium to map the coronary anatomy in patients with absolute contraindication of using conventional iodinated contrast agents, that can assist coronary physiology and IVUS for percutaneous management of CAD without any iodinated contrast use.

The incidence of iodine-induced thyrotoxicosis after administration of iodinated contrast agent remains low, which mostly results in subclinical hyperthyroidism in euthyroid patients regardless [1, 2]. Nevertheless, coexisting subclinical hyperthyroidism and ischemic heart disease combined with iodinate contrast load after CAG has a significant impact on worsening its clinical conditions that may require further treatment, clinical follow-up or even re-hospitalization after 1 year of CAG/PCI [3]. Bonelli et al. [3] analyzed an 810 


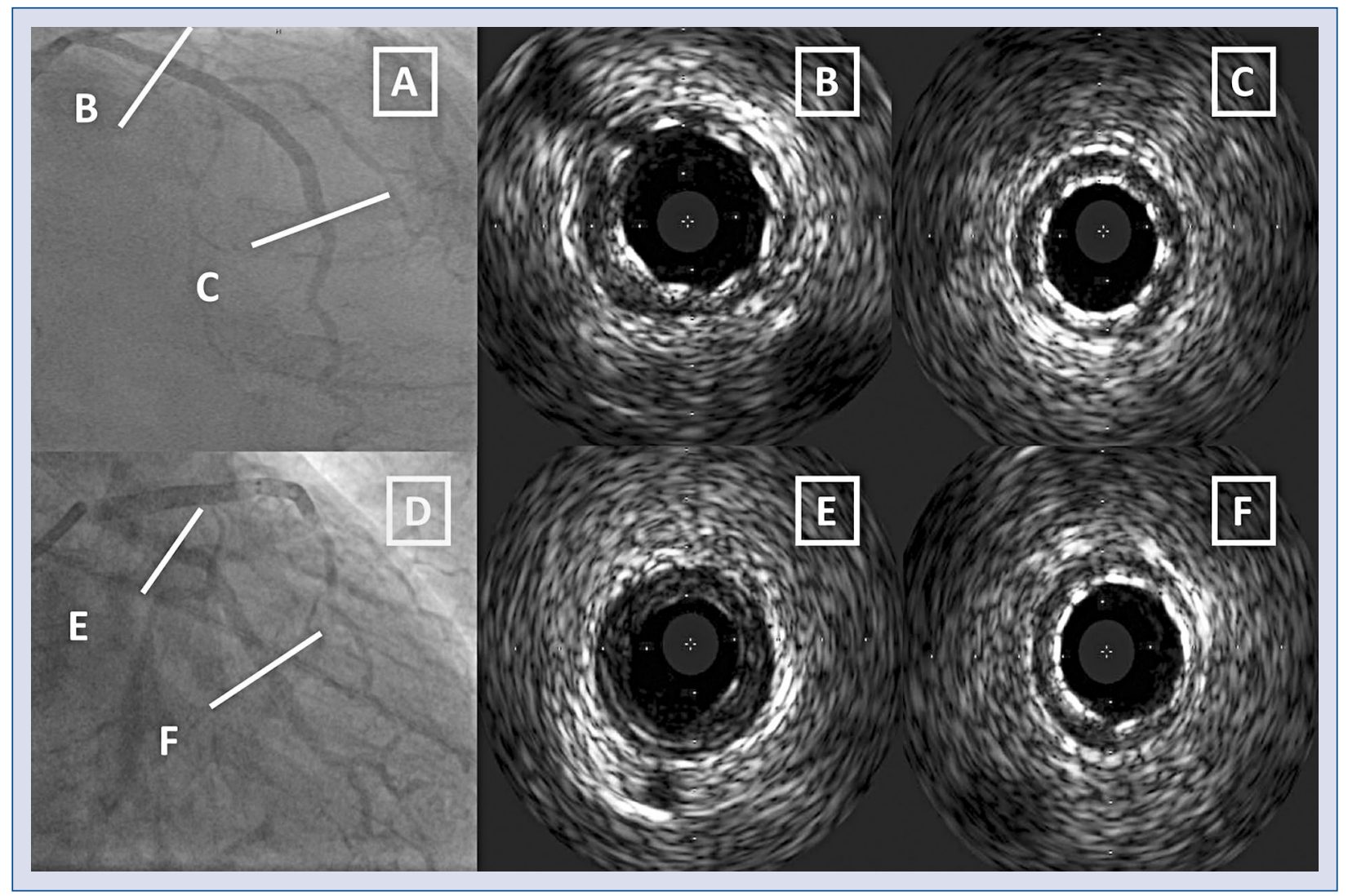

Figure 2. Images of intravascular ultrasound (IVUS) pullbacks performed in the left anterior descending artery (LAD), and the left circumflex artery (LCX); A-C. Pullback IVUS images of the LAD; D-F. Pullback IVUS images of the LCX.

patient cohort without known thyroid diseases who had undergone elective CAG. At baseline, $7.2 \%$ of the patients had hyperthyroidism, that increased to $10 \%$ after CAG. Independent predictors of hyperthyroidism development were baseline free $\mathrm{T}_{4}$ levels, thyroid nodules, age $>60$, male gender, and positive family history of thyroid diseases. Despite endocrinology follow up and therapy optimization, $4.2 \%$ at 3 months, and $2.5 \%$ at one year still had hyperthyroidism [3]. Additionally, in elderly patients, the risk of thyrotoxicosis after CAG with underlying thyroid disease is increased [9], and can be potentially life threatening, that may lead to cardiac tachyarrhythmias, heart failure, pulmonary arterial hypertension, pulmonary embolism, and cardiomyopathy.

Current guidelines propose early decision making on timing of angiography and possible intervention, especially in high-risk patient subgroups (elevated cardiac biomarkers at baseline, coexisting diabetes mellitus, Global Registry of Acute Coronary Events Risk Score [GRACE score > 140], age of $75 \leq$ years) with the ultimate goals of reducing length of hospital stay, refractory ischemia and mor- tality. In the present report, the patient's GRACE score was 137, thus current ESC/EACTS guidelines indicates invasive treatment in $<72$ hours (Class I, Level A) [10]. The use of coronary physiology (such as FFR or instant wave-free ratio) has been established as a feasible and effective option for culprit lesion identification in ACS patients with multivessel disease, nevertheless its prognostic value remains limited [10]. Moreover, identification of the culprit lesion remains challenging in a nonnegligible number of cases with non-ST elevation ACS, that may require additional tools — such as intravascular imaging - which may further assist clinical decision making. Coronary physiology combined with intravascular imaging (IVUS in the current report) provides a meticulous assessment of plaque instability and flow limitation, that has also confirmed the need for intervention in the aforementioned clinical scenario where conventional iodinated contrast use was not amenable.

Zero contrast and ultra-low contrast use [11] during PCIs treatment of complex CAD (such as chronic total occlusions) has been previously reported. The latter studies, however, reported 
contrast administration (zero contrast PCI was defined $<15 \mathrm{~mL}$ use of contrast medium), or recent diagnostic angiography was available before PCI that served as a road map for these technically challenging cases.

The use of gadolinium, as an alternative option for replacing iodinated contrast media for coronary angiography has been previously introduced [8], however its use has been limited due to (a) lower image quality, (b) potential adverse events of ventricular tachycardia/fibrillation or hemodynamic instability, and (c) its relatively high cost. The combination of IVUS and gadolinium has been reported to overcome an adverse reaction of iodinated contrast induced thrombocytopenia [7].

Applying gadolinium only for coronary road mapping (limiting its use only for the initial and final angiography) in combination with coronary physiology and IVUS is a potential option to guide complex coronary interventions with the benefit of fully avoiding using low osmolality iodinated contrast agents and hindering nephrotoxicity, adverse allergic reactions or thyrotoxicosis post-PCI. Before gadolinium injection, precautionary steps are mandatory to avoid adverse cardiac events. Herein, protocol included the following steps: (a) administration of intravenous beta-blocker, (b) applying defibrillator paddles, (c) monitoring arterial pressure, (d) avoiding pressure dampening of guiding catheters (use of guiding catheters with side holes however, could further reduce image quality), (e) and adding slow and low amounts of test injections.

\section{Conclusions}

This report demonstrates the feasibility of a concept combining gadolinium, IVUS, and coronary physiology to treat patients with CAD and severe coexisting comorbidities that can be aggravated by iodinated contrast media.

Conflict of interest: Kambis Mashayekhi, MD: honoraria/consulting fees from Ashai Intecc, Boston, Medtronic, Teleflex, Cardinal Health, Abboth, Biotronik, Terumo, AstraZeneca, Daiichi Sankyo; Remaining authors: nothing to disclose.

\section{References}

1. Marraccini P, Bianchi M, Bottoni A, et al. Prevalence of thyroid dysfunction and effect of contrast medium on thyroid metabolism in cardiac patients undergoing coronary angiography. Acta Radiol. 2013; 54(1): 42-47, doi: 10.1258/ar.2012.120326, indexed in Pubmed: 23125395.

2. Hintze G, Blombach O, Fink H, et al. Risk of iodine-induced thyrotoxicosis after coronary angiography: an investigation in 788 unselected subjects. Eur J Endocrinol. 1999; 140(3): 264-267, doi: 10.1530/eje.0.1400264, indexed in Pubmed: 10216523.

3. Bonelli N, Rossetto R, Castagno D, et al. Hyperthyroidism in patients with ischaemic heart disease after iodine load induced by coronary angiography: Long-term follow-up and influence of baseline thyroid functional status. Clin Endocrinol (Oxf). 2018; 88(2): 272-278, doi: 10.1111/cen.13494, indexed in Pubmed: 29023926.

4. Rieger J, Sitter T, Toepfer M, et al. Gadolinium as an alternative contrast agent for diagnostic and interventional angiographic procedures in patients with impaired renal function. Nephrol Dial Transplant. 2002; 17(5): 824-828, doi: 10.1093/ndt/17.5.824, indexed in Pubmed: 11981070.

5. Sayin T, Turhan S, Akyürek O, et al. Gadolinium:nonionic contrast media (1:1) coronary angiography in patients with impaired renal function. Angiology. 2007; 58(5): 561-564, doi: 10.1177/0003319707303640, indexed in Pubmed: 17906283.

6. Juneman E, Saleh L, Thai $\mathrm{H}$, et al. The use of gadolinium in patients with contrast allergy or renal failure requiring coronary angiography, coronary intervention, or vascular procedure. Catheter Cardiovasc Interv. 2011; 78(5): 747-754, doi: 10.1002/ ccd.22907, indexed in Pubmed: 21780275.

7. Cubero-Gómez J, Márquez FG, la-Llera LDd, et al. Severe thrombocytopenia induced by iodinated contrast after coronary angiography: The use of gadolinium contrast and intravascular ultrasound as an alternative to guide percutaneous coronary intervention. Rev Port Cardiol. 2017; 36(1): 61.e1-61.e4, doi: 10.1016/j.repce.2016.12.009.

8. Kälsch H, Kälsch T, Eggebrecht H, et al. Gadolinium-based coronary angiography in patients with contraindication for iodinated x-ray contrast medium: a word of caution. J Interv Cardiol. 2008; 21(2): 167-174, doi: 10.1111/j.1540-8183.2007.00340.x, indexed in Pubmed: 18312304.

9. Ledingham D, Carey P, Junejo S. The dangers of iodine-based contrasts in an elderly patient with thyroid disease. BMJ Case Rep. 2015; 2015, doi: 10.1136/bcr-2014-207657, indexed in Pubmed: 25804944 .

10. Neumann FJ, Sousa-Uva M, Ahlsson A, et al. 2018 ESC/EACTS Guidelines on myocardial revascularization. Eur Heart J. 2019; 40(2): 87-165, doi: 10.1093/eurheartj/ehy394, indexed in Pubmed: 30165437.

11. Ali ZA, Karimi Galougahi K, Nazif T, et al. Imaging- and physiology-guided percutaneous coronary intervention without contrast administration in advanced renal failure: a feasibility, safety, and outcome study. Eur Heart J. 2016; 37(40): 3090-3095, doi: 10.1093/eurheartj/ehw078, indexed in Pubmed: 26957421. 
